\title{
ON A PROBLEM OF A. KUROSCH
}

\section{J. LEVITZKI}

The following problem was posed by Kurosch [2]:1 Suppose that an algebraic algebra $A$ over a field $F$ has a finite number of generators over $F$, and suppose further that $A$ is of bounded degree over $F$. Is then $A$ of finite dimensionality over $F$ ? Kurosch answered his problem in the affirmative in the case where the elements of the algebra are of degree not greater than 3. As was observed by N. Jacobson [1], Kurosch's problem is equivalent to the following: Is any algebraic algebra of bounded degree locally finite ${ }^{3}$ over $F$ ? Jacobson succeeded in reducing this problem to the special case of nil-algebras. In the present note we supplement Jacobson's results by proving that any nil-algebra of bounded index (degree) is locally finite. Jacobson's results combined with this theorem answer in the affirmative the question raised by Kurosch.

To obtain this result we make use of the notion of semi-nilpotency ${ }^{4}$ and of its counterpart, the semi-regularity, which were introduced by the author in a previous paper [3]. As may be easily verified, each finitely generated nilpotent algebra is of finite dimensionality, and conversely, each nil-algebra of finite dimensionality is nilpotent. Hence, for nil-algebras the notion of local finiteness coincides with the notion of semi-nilpotency. Thus the above formulated result concerning nil-algebras is a consequence of the following more general theorem concerning nil-rings which will be proved in this paper: Each nil-ring of bounded index is semi-nilpotent. It is of interest to note in this connection that an analogous theorem does not hold for associative multiplicative systems (semi-groups). ${ }^{5}$

It will be convenient to adopt the terminology introduced by the author in [3]. Thus we use here the term radical to denote the sum $N(S)$ of all two-sided semi-nilpotent ideals of a ring $S$. We shall need the following theorems which were proved in [3]:

Received by the editors July 6, 1946.

1 Numbers in brackets refer to the bibliography at the end of the paper.

2 For the terminology compare Jacobson [1].

${ }^{3}$ An algebra $A$ is called locally finite over $F$ if each finite set of elements of $A$ generates an algebra of finite dimensionality over $F$.

4 A ring is called semi-nilpotent if each finite set of elements in this ring generates a nilpotent ring. A ring which is not semi-nilpotent is called semi-regular.

${ }^{5}$ Compare Kurosch [2, proof of Theorem 5]. This was observed independently also by Dr. Th. Motzkin of Jerusalem. 
1. The radical $N(S)$ is a semi-nilpotent two-sided ideal which contains all one-sided semi-nilpotent ideals of the ring.

2. The radical contains all nilpotent ideals of the ring.

3. The radical of the quotient-ring $S / N(S)$ is zero.

4. If the radical of a ring $S$ is zero, and if $a$ is a nonzero element of $S$, then $a \cdot S$ and $S \cdot a$ are semi-regular rings.

Definition. An integer $n=n(S)$ is called the upper index of a nilring $S$, if $S$ contains elements $a$ whose index is $n$ (that is, $a^{n}=0$, $\left.a^{n-1} \neq 0\right)$, but it does not contain elements of higher index. Such an integer $n$ exists of course for each nil-ring of bounded index.

LEMMA. If $S$ is a semi-regular nil-ring of bounded index, then there exists a semi-regular nil-ring whose upper index is smaller than the upper index of $S$.

Proof. Let $N(S)$ be the radical of $S$, then by 3 the radical of the ring $T=S / N(S)$ is zero. Since $S$ is semi-regular, it follows that $S \neq N(S)$, and hence that $T$ is a semi-regular nil-ring of bounded index so that $n(T) \leqq n(S)$. In the case where $n(T)<n(S)$ the lemma is proved. Thus it remains to deal with the case $n(T)=n(S)$. Now let $a$ be an element of $T$ so that $a \neq 0, a^{2}=0$, and let us consider the right ideal $U=a \cdot T$ of $T$. The ring $U$ is by 3 and 4 also semi-regular, and clearly $n(U) \leqq n(T)$. Again in the case where $n(U)<n(T)$ the lemma is proved, and thus it remains to deal with the case where $n(U)=n(T)$. In this case the ring $U$ contains an element $b$ whose index is $n=n(T)$, that is, $b^{n-1} \neq 0$, and since $U=a T$ we may put $b=a \cdot t, t \in T$. We consider now the following two cases:

Case 1. $b^{n-1} U \neq 0$. Then $b^{n-1} a \neq 0$, and by putting $c=a+b$, it follows on account of $a^{2}=0$ that $c^{n}=(a+b)^{n}=(a+a t)^{n}=(a t)^{n-1} a=b^{n-1} a \neq 0$, which is a contradiction, since $n$ is the upper index of $T$.

Case 2. $b^{n-1} U=0$ for each element $b$ of $U$. In this case denote by $M$ the set of all elements $x$ of $U$ satisfying the relation $x U=0$, then

$$
b^{n-1} \in M, \quad b \in U .
$$

Since $M^{2} \subseteq M U=0$, it follows that $M$ is a nilpotent ideal in $U$, and hence by 1 and 2 we have

$$
N(U) \supseteq M
$$

where $N(U)$ is the radical of $U$. Now let us consider the quotient-ring $V=U / N(U)$, which is also a semi-regular nil-ring of bounded index. 
Since further by (1) all $(n-1)$ th powers of the elements of $U$ belong to $M$, it follows by (2) that for each $v$ of $V$ we have $v^{n-1}=0$, which implies that the upper index of the semi-regular ring $V$ is smaller than $n(S)$. This concludes the proof of the lemma.

THEOREM. Each nil-ring $S$ of bounded index is semi-nilpotent.

Proof. Suppose that $S$ is semi-regular, then it would follow by the foregoing lemma that there exists a semi-regular nil-ring with an upper index 1 , which is self-contradictory.

Added in Proof. The problem of Kurosch was also treated by Irving Kaplansky (On a problem of Kurosch and Jacobson, Bull. Amer. Math. Soc. vol. 52 (1946) pp. 496-500). In this paper Kaplansky succeeds in solving, in conjunction with results of Jacobson, the problem of Kurosch under the restriction that the number of elements in the coefficient field is at least equal to $n$, where $n$ is a common bound for the degrees of the elements in the algebra.

\section{BIBLIOGRAPHY}

1. N. Jacobson, Structure theory for algebraic algebras of bounded degree, Ann. of Math. vol. 46 (1945) pp. 695-707.

2. A. Kurosch, Ringtheoretische Probleme die mit dem Burnsideschen Problem ueber periodische Gruppen in Zusammenhang stehen, Bull. Acad. Sci. URSS. Sér. Math. vol. 5 (1941) pp. 233-240.

3. J. Levitzki, On the radical of a general ring, Bull. Amer. Math. Soc. vol. 49 (1943) pp. 461-466.

Hebrew UnIVERSITY 